11

\title{
Волноводные устройства на основе линейных дефектов в металлических электромагнитных кристаллах
}

\author{
() А.Ю. Ветлужский
}

Институт фризического материаловедения СО РАН, 670047 Улан-Удэ, Россия

e-mail: vay@ipms.bscnet.ru

(Поступило в Редакцию 25 февраля 2016 г. В окончательной редакции 10 мая 2016 г.)

Рассмотрена работа волноводов, представляющих собой линейные дефекты в двумерных металлических фотонных кристаллах. Показана возможность канализации излучения такими структурами на частотах первой разрешенной зоны. Обсужден физический механизм, приводящий к локализации излучения в области дефектов в кристалле.

DOI: 10.21883/JTF.2017.01.44033.1775

\section{Введение}

Одним из интересных и перспективных направлений современной радиофизики является разработка и изучение свойств фотонных (или электромагнитных) кристаллов и метаматериалов. Этими терминами в настоящее время обозначают разные объекты, обладающие тем не менее во многом сходными чертами. В первую очередь, это периодический характер их внутренней структуры, представляющей собой чередование диэлектрических или металлических элементов с различной геометрией и разными электрофизическими характеристиками и обусловливающей особенности взаимодействия с ними электромагнитных излучений.

Говоря об этих особенностях, для фотонных кристаллов в первую очередь следует отметить наличие зонной структуры спектра собственных электромагнитных состояний таких объектов, выражающееся в формировании диапазонов частот, в которых излучение либо свободно проходит через кристалл (разрешенные зоны), либо интенсивно подавляется (запрещенные зоны) [1]. Кроме того, большой интерес вызывает наличие у фотонных кристаллов ярко выраженной дисперсии, причем как частотной, так и пространственной, что открывает широкие возможности для их весьма разнообразных практических приложений [2]. Применительно к метаматериалам основной интерес в подобных структурах вызывает необычный характер отражений и преломлений волн на их границах, вызванный аномальными - в том числе и отрицательными - значениями эффективных проницаемостей таких структур, с чем, например, связываются возможные существенные изменения в технике передачи оптических изображений, в частности, преодоление дифракционного предела [3].

Принципиальное различие между искусственными кристаллами и метаматериалами заключается в масштабе пространственных неоднородностей, образующих их внутреннюю структуру, - для первых он сопоставим с длиной волны излучения, для вторых - много меньше ее. Это определяет требования, с одной стороны, к технологии изготовления таких материалов, в частности, для кристаллов - к точности выполнения и размещения отдельных элементов [4], с другой - к диапазонам частот, на которых они могут быть использованы на практике уже в настоящее время.

Oсобое место среди таких структур занимают металлические искусственные кристаллы - периодические решетки, образованные элементами, имеющими высокую проводимость в радио- и инфракрасных диапазонах. Первые работы, посвященные изучению их свойств, появились еще в середине прошлого века и были основаны на проведении аналогии со свойствами плазменных образований, о чем позволяло говорить наличие пороговой частоты, ниже которой кристалл полностью подавляет распространение электромагнитного излучения, а выше - становится „прозрачным“ для него. Так, в [5] получено выражение, связывающее значение плазменной частоты двумерного металлического кристалла с его геометрическими параметрами, которое неоднократно уточнялось в последние годы [6] в связи с ростом интереса к электродинамическим свойствам подобных структур.

Таким образом, металлические кристаллы с полным основанием могут быть отнесены как к фотонным кристаллам, так и метаматериалам. Наличие низкочастотной запрещенной зоны позволяет говорить о них, как о сплошных средах с отрицательной диэлектрической проницаемостью, обусловливающей подавление излучения вплоть до плазменной частоты, при этом физический механизм этого подавления не связан с брэгговским отражением, являющимся причиной формирования запрещенных зон в периодических структурах. Важным представляется то, что на частотах выше плазменной металлические кристаллы также проявляют необычные электрофизические свойства. В работе [7] на основе анализа резонансных эффектов в таких структурах показано, что в первой разрешенной зоне возможно рассмотрение металлического кристалла как сплошной сильно диспергирующей среды, показатель преломления которой меныше единицы. На более высоких частотах, 

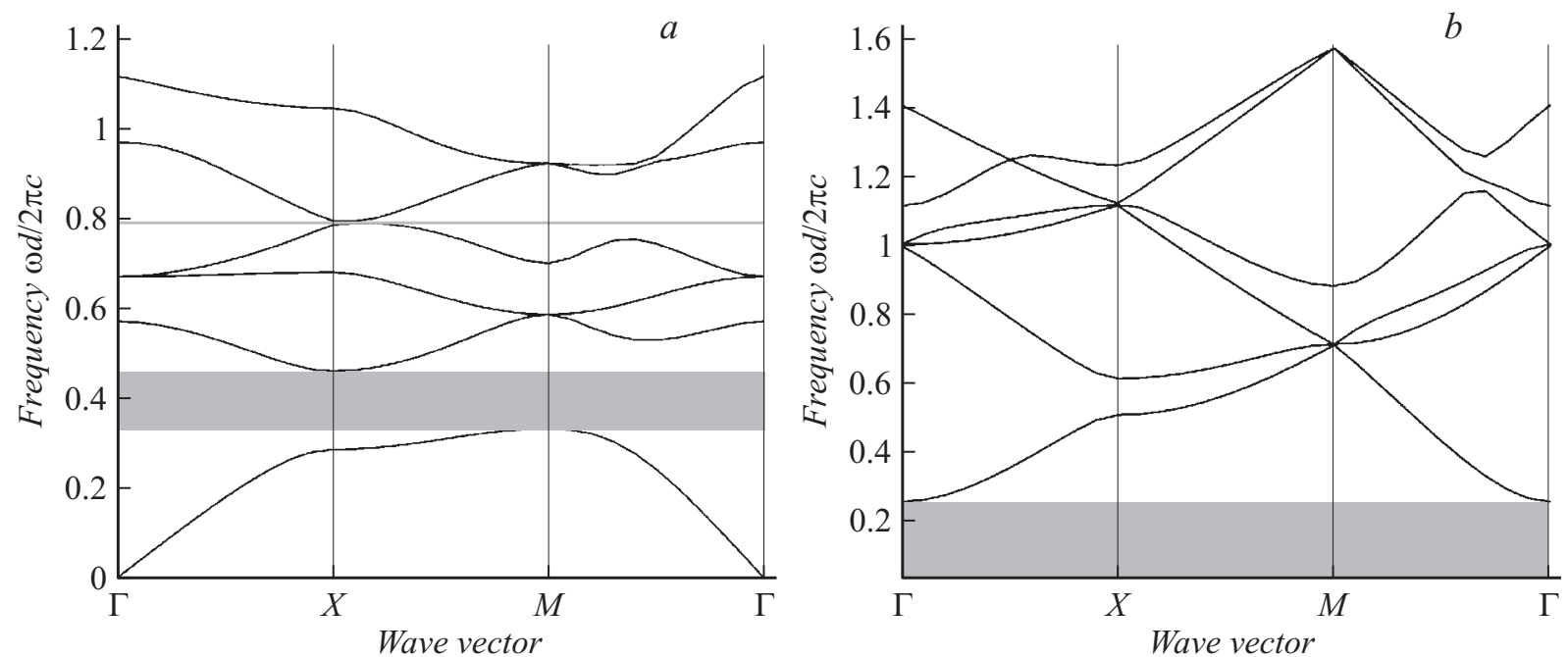

Рис. 1. Дисперсионные диаграммы, показывающие наличие полных запрещенных зон в рассматриваемой полосе частот для диэлектрического $(a)$ и металлического $(b)$ фотонных кристаллов.

при выполнении условия $d / \lambda>0.5$, где $d-$ период структуры, $\lambda$ - длина волны, наблюдается дальнейшее чередование запрещенных и разрешенных диапазонов частот, обусловленных уже типичной для фотонных структур брэгговской интерференцией. При этом в силу достаточно большого относительного периода структуры на этих частотах говорить о таких кристаллах как о сплошной среде становится уже некорректно.

Говоря о перспективах практического применения фотонных кристаллов, нужно сказать, что на настоящий момент они формулируются весьма разнообразно. Однако исторически первой и по сей день не теряющей актуальности следует, по-видимому, считать возможность создания на их основе волноведущих и резонаторных устройств различного типа и назначения. Общим для них является формирование линейных либо точечных дефектов в периодических структурах, выступающих в качестве областей локализации излучения, которая происходит за счет непрозрачности остального объема кристалла на частотах, соответствующих запрещенной зоне.

В настоящей работе мы рассмотрим возможность создания волноведущих систем на основе двумерных металлических фотонных кристаллов, функционирующих не только на частотах, соответствующих запрещенной, но и в первой разрешенной зоне. Основанием для этого служат предположения о возможности локализации излучения в дефектах кристалла за счет полного внутреннего отражения волн на границе с материалом с низким значением показателя преломления.

\section{Постановка задачи. Методика расчетов}

Рассмотрим двумерно-периодическую квадратную решетку с периодом $d$, образованную цилиндрами кругового поперечного сечения радиуса $a$, возбуждаемую
$E$-поляризованной волной (вектор $E$ параллелен осям цилиндров). Будем анализировать свойства таких структур, образованных металлическими (алюминий) и диэлектрическими (оксид алюминия $\mathrm{Al}_{2} \mathrm{O}_{3}$ ) элементами. Коэффициент заполнения $f=\pi a^{2} / d^{2}$ металлической структуры положим равным 0.008 (период $1 \mu \mathrm{m}$, радиус $0.05 \mu \mathrm{m}$ ), для диэлектрической -0.13 (радиус $0.2 \mu \mathrm{m}$ при том же периоде). Для описания диэлектрических свойств металла воспользуемся дисперсионной моделью Друдэ:

$$
\varepsilon^{\prime}(\omega)=1-\frac{\omega_{p}^{2}}{\omega^{2}+\gamma^{2}}, \quad \varepsilon^{\prime \prime}(\omega)=\frac{\omega_{p}^{2} \gamma}{\omega^{3}+\omega \gamma^{2}},
$$

где $\varepsilon^{\prime}(\omega)$ и $\varepsilon^{\prime \prime}(\omega)$ - действительная и мнимая части диэлектрической проницаемости, $\omega_{p}$ - плазменная частота, $\gamma$ - факторы затухания, равные для алюминия соответственно 3570 и $19.4 \mathrm{THz}$ [8]. Диэлектрическую проницаемость оксида алюминия примем равной 9.5, потерями в нем на рассматриваемых в работе частотах пренебрежем.

Для определения зонной структуры спектра пропускания построим дисперсионные диаграммы, характеризующие модовый состав излучения, проходящего через пространственно неограниченный кристалл. Для этого воспользуемся методом разложения по плоским волнам (plane wave expansion method) для диэлектрической структуры и его модифицированным вариантом [9] для определения свойств металлического кристалла. На рис. 1 представлены соответствующие зависимости, на которых по оси ординат отложены значения относительной частоты $\omega d / 2 \pi c$, где $\omega-$ циклическая частота, $c$ - скорость света, а по оси абсцисс - основные симметричные точки первой зоны Бриллюэна двумерной квадратной решетки. Количество плоских волн, использованных при построении зонной структуры, для диэлектрической решетки - 121, для металлической 197. Видно, что в рассмотренном диапазоне в обоих 

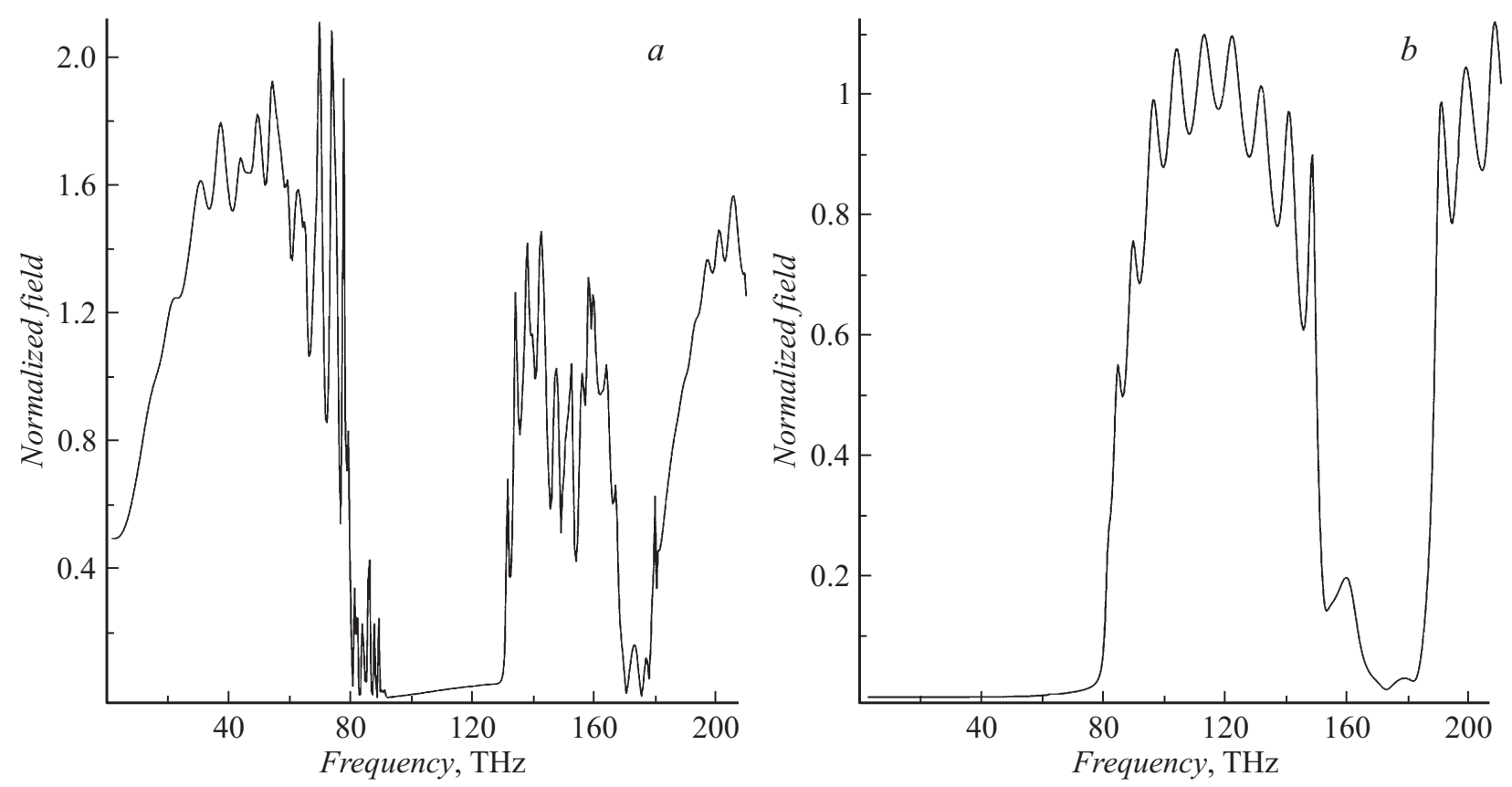

Рис. 2. Спектры пропускания диэлектрического (a) и металлического $(b)$ фотонных кристаллов.

случаях имеются полные запрещенные зоны (отмечены на рисунке), в пределах которых излучение не распространяется в кристалле ни в одном направлении в плоскости, перпендикулярной осям цилиндров. При этом для заданных параметров в спектре пропускания металлического кристалла имеется единственная такая зона, располагающаяся в области низких частот.

Дальнейший анализ проводился для кристаллов, облучаемых плоской волной, падающей на структуру в направлении ГХ или - с использованием индексов Миллера - в направлении $|10|$ относительно соответствующей кристаллографической плоскости. Расчеты выполнялись методом самогласованных уравнений (selfconsistent equation method) [10], весьма эффективным при решении подобных задач.

\section{Анализ и обсуждение результатов}

На рис. 2 изображены частотные зависимости относительного уровня поля, прошедшего через диэлектрическую и металлическую структуры, состоящие из 121 элемента (11 на 11), и определяемого вблизи их границ. В рассмотренном диапазоне в обоих случаях наблюдается чередование полос пропускания и запирания излучения, соответствующих зонным диаграммам на рис. 1. Эти же данные указывают на то, что вторая запрещенная зона для металлического кристалла не является полной, т.е. подавление распространения излучения происходит только вдоль направления ГХ в структуре.

Создадим линейный дефект в кристаллах, удалив центральный ряд элементов вдоль направления распространения волны. Очевидно, что образованный канал сможет при определенных условиях функционировать в качестве волновода. Для этого необходимо, чтобы его „стенки“ были непрозрачны для излучения в соответствующем частотном диапазоне.

Из рис. 2 определим наиболее характерные частоты, на которых выполним анализ пространственной структуры поля в дефектном кристалле. Для диэлектрической структуры рассмотрим распределение поля на частотах 75, 110 и $140 \mathrm{THz}$. Первая относится к первой разрешенной, вторая - к первой запрещенной и, наконец, третья - ко второй разрешенной зонам. Для металла построим такие картины на частотах 80, 110 и $175 \mathrm{THz}$. Первая из них располагается в пределах полной запрещенной зоны, вторая - в первой разрешенной зоне, а последняя - во второй (неполной) запрещенной зоне. Отметим, что указанные частоты существенно меньше плазменной частоты металла, поэтому дисперсионные свойства материала элементов в рассматриваемом диапазоне сказываются лишь в плавном и незначительном по величине изменении диэлектрической проницаемости. Из этого следует, что все дальнейшие выводы будут также справедливы в отношении подобных структур, функционирующих на более низких частотах.

На рис. 3 и 4 представлены картины распределения поля в кристаллах, состоящих из диэлектрических и металлических цилиндров соответственно.

Рис. 3, a, с демонстрирует, что на частотах, относящихся к разрешенным зонам, волноводного распространения излучения по сформированному каналу не возникает. Более того, поле частично локализуется в пределах бездефектных областей кристалла, находящихся по обе стороны от канала. Это, по-видимому, связано с интерференцией волн, дифрагировавших на границах 

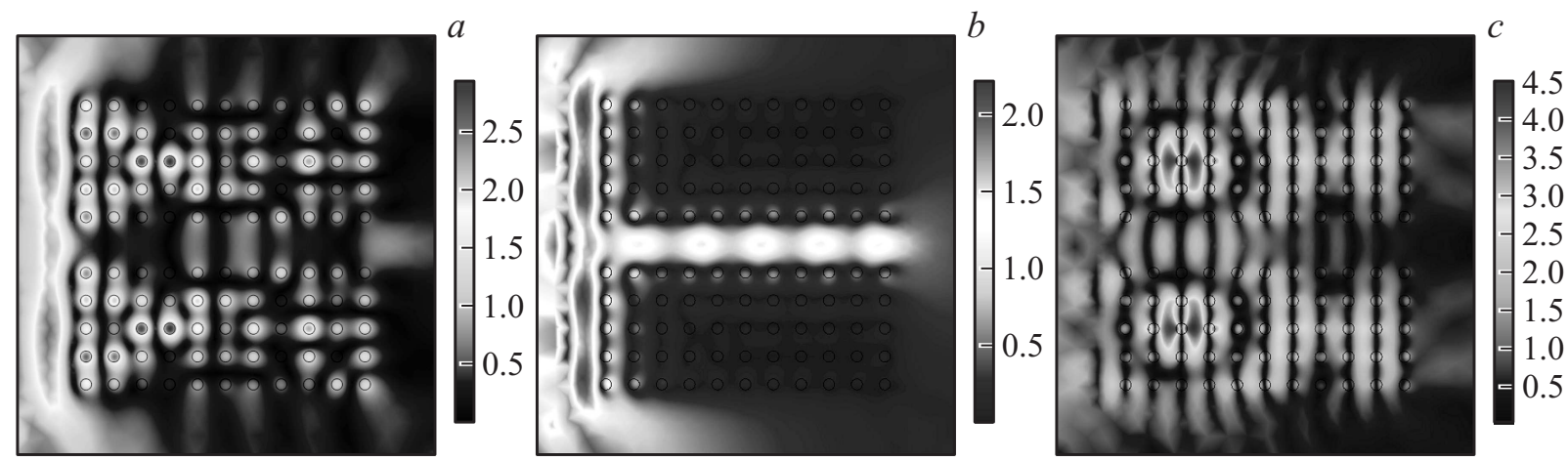

Рис. 3. Пространственное распределение поля в диэлектрическом фотонном кристалле на частотах 75 (a), $110(b)$ и $140 \mathrm{THz}(c)$.
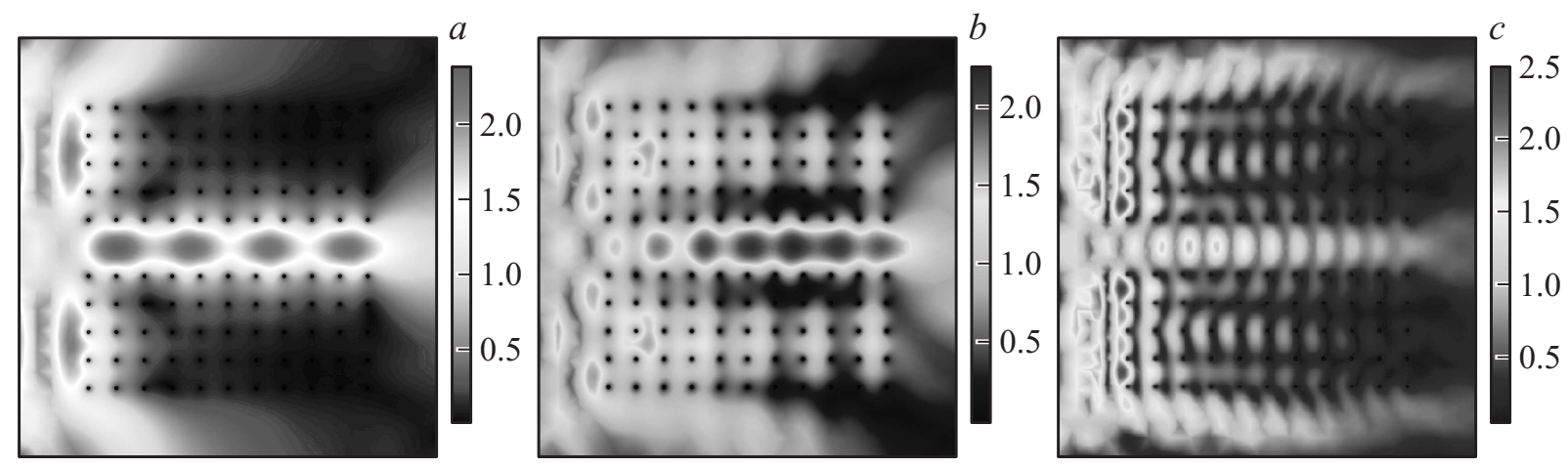

Рис. 4. Пространственное распределение поля в металлическом фотонном кристалле на частотах $80(a), 110(b)$ и $175 \mathrm{THz}(c)$.
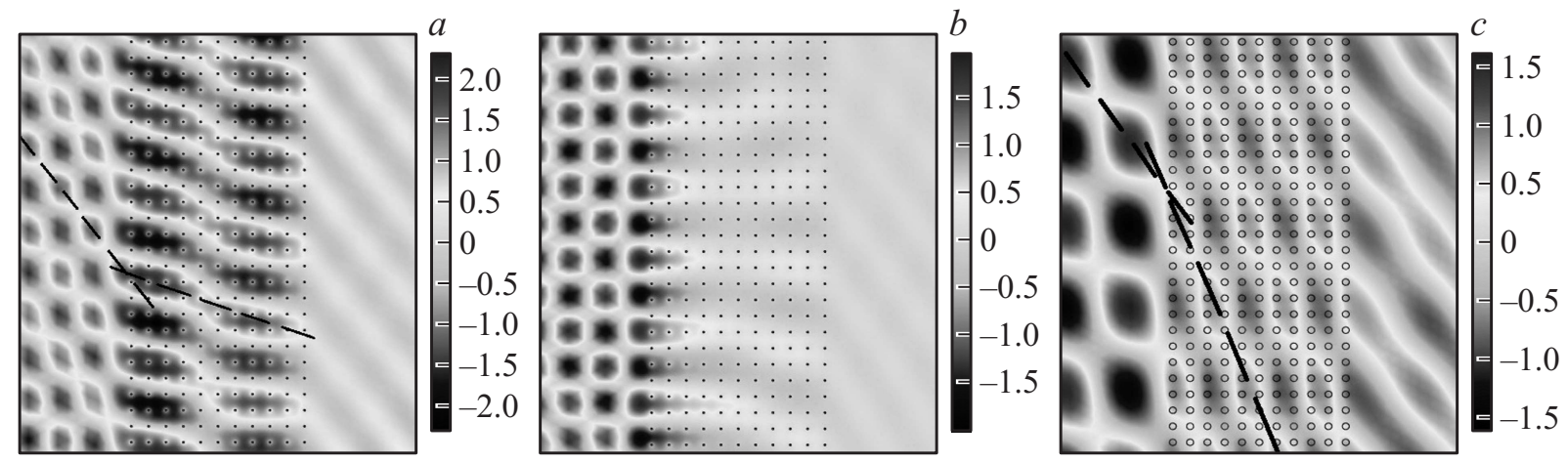

Рис. 5. Пространственное распределение поля при наклонном падении волны на границу кристалла: $a$ - металлическая структура, угол падения $35^{\circ}$, частота $110 \mathrm{THz}, b-$ металлическая структура, угол падения $42^{\circ}$, частота $110 \mathrm{THz} ; c-$ диэлектрическая структура, угол падения $35^{\circ}$, частота $50 \mathrm{THz}$.

указанных областей. На рис. $3, b$ изображена типичная картина локализации излучения в волноведущем канале на частоте, соответствующей полной запрещенной зоне.

Рис. 4, $a$ также демонстрирует эффективную канализацию излучения вдоль линейного дефекта в металлическом кристалле, непрозрачном для излучения. Отметим, что понижение частоты ниже $70 \mathrm{THz}$ приводит к отсечке распространения волн по данному волноводу, поскольку ширина канала оказывается недостаточна для возбуждения в нем основной волноводной моды.

Наибольший интерес представляют результаты на pис. 4 (b). Поскольку рассматриваемая частота $110 \mathrm{THz}$ соответствует разрешенной зоне, видно, что излучение свободно (с незначительным отражением) проходит через левую - облучаемую - границу кристалла по всей его ширине. Однако по мере распространения в глубь структуры происходит перераспределение поля. Рассеиваясь элементами, часть излучения локализуется в волноводном канале и далее распространяется, не выходя за его пределы. В результате на выходе из структуры поле оказывается в основном сосредоточено в пределах этого канала. При этом эффективность такого волновода оказывается не ниже, чем при его работе на частотах запрещенной зоны. Это может быть 
объяснено тем, что в запрещенной зоне возбуждение волновода происходит только при падении излучения на границу структуры, при этом бо́льшая часть энергии падающей волны кристаллом отражается. На частотах разрешенной зоны из-за малого отражения в кристалл поступает бо́льшая энергия поля, а ее затекание в волновод происходит по всей его длине.

Рис. 4, , соответствующий частоте $175 \mathrm{THz}$, иллюстрирует работу волновода во второй запрещенной зоне. Очевидна его крайне низкая эффективность, связанная, в первую очередь, с достаточно высокой пропускающей способностью кристалла, поскольку подавление распространения волн в нем происходит только в направлении ГХ и близких к нему (рис. $1, b$ ), а следовательно, энергия, переносимая волной, движущейся по волноводному каналу, частично перераспределяется в окружающий объем кристалла. С другой стороны, падающая в направлении ГХ волна практически не проникает в кристалл, что исключает возможность дополнительного притока энергии в канал за счет дифракции на элементах структуры, как это наблюдалось в предыдущем случае.

В целом представленные результаты показывают, что использование в качестве волноводов линейных дефектов в металлических кристаллах на частотах не только запрещенной, но и первой разрешенной зоны позволяет существенно расширить рабочую полосу частот таких устройств.

Для однозначной трактовки физического механизма, приводящего к канализации излучения в дефектном канале на частотах разрешенной зоны, рассмотрим наклонное падение волн на металлический и диэлектрический кристаллы. На рис. 5 представлены картины преломления волн при взаимодействии с границей двумерной структуры.

Рис. 5, $a$ описывает ситуацию, при которой волна с частотой $110 \mathrm{THz}$ падает под углом $35^{\circ}$ на металлический кристалл. Штриховыми линиями на рисунке отмечены поверхности волновых фронтов падающей и прошедшей в структуру волн. Очевидно, что угол преломления $\left(\sim 66^{\circ}\right)$ в данном случае превышает угол падения, а следовательно, рассматривая кристалл, как некоторую среду с эффективным показателем преломления, можно утверждать, что его величина в данном случае меньше единицы, т.е. в структуре распространяются быстрые по отношению к окружающему пространству волны. Основанием для описания свойств кристалла как сплошной среды в данном случае может служить тот факт, что характер волнового фронта, типичный для плоской волны, не нарушается при прохождении через границу из-за дискретности кристалла.

На рис. $5, b$ изображено распределение поля при увеличении угла падения до $42^{\circ}$. Представленная картина соответствует выполнению условий полного внутреннего отражения волны при ее переходе из оптически более плотной среды в менее плотную. Видно, что излучение практически не проходит через кристалл, локализуясь вблизи его границы и приобретая характер поверхностной волны.
И, наконец, на рис. 5, с изображена аналогичная картина преломления волны на границе с диэлектрическим кристаллом на частоте $50 \mathrm{THz}$, т.е. в первой разрешенной зоне. Отмеченные штрихом поверхности волновых фронтов указывают на обычный характер преломления на границе воздух-диэлектрик.

Таким образом, представленные результаты позволяют однозначно интерпретировать физический механизм, приводящий к локализации излучения в дефектном канале в металлическом кристалле, как полное внутреннее отражение волн на „стенках“ волновода.

\section{Заключение}

В настоящей работе показана возможность канализации излучения в линейных дефектах металлических фотонных кристаллов на частотах полной запрещенной и первой разрешенной зон. Доказано, что в последнем случае физический механизм, приводящий к локализации излучения в волноведущем канале, заключается в полном внутреннем отражении волн при падении на границу среды с показателем преломления, меньшим единицы.

Работа выполнена при финансовой поддержке РФФИ в рамках научного проекта № 15-47-04315.

\section{Список литературы}

[1] Joannopoulos J.D. Photonic Crystals: Molding the Flow of Light. Princeton Univ. Press, 2008. 304 p.

[2] Simovski C., Belov P., Atrashchenko A., Kivshar Y. // Advan. Mater. 2012. Vol. 24. P. 4229-4248.

[3] Гуляев Ю.В., Лагарьков А.Н., Никитов С.А. // Вестн. РАН. 2008. T. 78. № 5. C. 438-449.

[4] Topolancik J., Ilic B., Vollmer F. // Phys. Rev. Let. 2007. V01. 99. P. 253901.

[5] Brown J. // Proc. Inst. Elect. Eng. (London). 1953. Part IV. Vol. 100. Monograph № 62R. P. 51-62.

[6] Tretyakov S.A. Analytical Modeling in Applied Electromagnetics. Artech House: Norwood, MA, 2003.

[7] Ветлужский А.Ю. // Журн. радиоэлектрон. 2015. № 1. Электронный ресурс. Режим доступа: http://jre.cplire.ru/ jre/jan15/index.html.

[8] El-Kady I. et al. // Phys. Rev. B. 2000. Vol. 62. N 23. P. 15 299-15302.

[9] Kuzmiak V., Maradudin A., Pincemin F. // Phys. Rev. B. 1994. Vol. 50. N 23. P. $16835-16844$.

[10] Ветлужсский А.Ю. // ЖЭТФ. 2009. Т. 136. № 2. С. 356-361. 